\title{
Overexpression of Snail is associated with lymph node metastasis and poor prognosis in patients with gastric cancer
}

\author{
Na Ri Shin ${ }^{1,4 \dagger}$, Eun Hui Jeong ${ }^{5 \dagger}$, Chang In Choi ${ }^{3,4 \dagger}$, Hyun Jung Moon ${ }^{1,4}$, Chae Hwa Kwon ${ }^{1,4}$, In Sun Chu ${ }^{6}$, \\ Gwang Ha Kim ${ }^{2,4}$, Tae Yong Jeon ${ }^{3,4}$, Dae Hwan Kim, ${ }^{3,4}$, Jae Hyuk Lee ${ }^{5}$ and Do Youn Park ${ }^{1,4^{*}}$
}

\begin{abstract}
Background: Epithelial-mesenchymal transition (EMT) plays a significant role in tumor progression and invasion. Snail is a known regulator of EMT in various malignant tumors. This study investigated the role of Snail in gastric cancer.

Methods: We examined the effects of silenced or overexpressed Snail using lenti-viral constructs in gastric cancer cells. Immunohistochemical analysis of tissue microarrays from 314 patients with gastric adenocarcinoma (GC) was used to determine Snail's clinicopathological and prognostic significance. Differential gene expression in 45 GC specimens with Snail overexpression was investigated using CDNA microarray analysis.

Results: Silencing of Snail by shRNA decreased invasion and migration in GC cell lines. Conversely, Snail overexpression increased invasion and migration of gastric cancer cells, in line with increased VEGF and MMP11. Snail overexpression ( $\geq 75 \%$ positive nuclear staining) was also significantly associated with tumor progression $(P<0.001)$, lymph node metastases $(P=0.002)$, lymphovascular invasion $(P=0.002)$, and perineural invasion $(P=0.002)$ in the $314 \mathrm{GC}$ patients, and with shorter survival $(P=0.023)$. CDNA microarray analysis revealed 213 differentially expressed genes in GC tissues with Snail overexpression, including genes related to metastasis and invasion.
\end{abstract}

Conclusion: Snail significantly affects invasiveness/migratory ability of GCs, and may also be used as a predictive biomarker for prognosis or aggressiveness of GCs.

Keywords: Stomach, Adenocarcinoma, Snail, Lymph node metastasis, Survival

\section{Background}

Epithelial-mesenchymal transition (EMT), a developmental process whereby epithelial cells reduce intercellular adhesion and acquire myofibroblastic features, is critical to tumor progression [1-3]. During EMT, significant changes occur, including downregulation of epithelial markers such as E-cadherin, translocation of $\beta$-catenin (i.e., dissociation

\footnotetext{
* Correspondence: pdy220@pusan.ac.kr

'Equal contributors

'Department of Pathology, Pusan National University Hospital and Pusan National University School of Medicine, 1-10 Ami-Dong, Seo-Gu, Busan 602-739, South Korea

${ }^{4}$ BioMedical Research Institute, Pusan National University Hospital, Busan, South Korea

Full list of author information is available at the end of the article
}

of membranous $\beta$-catenin and translocation into the nuclear compartment), and upregulation of mesenchymal markers such as vimentin and N-cadherin [3-6]. EMT is induced by repression of E-cadherin expression by EMT regulators such as Snail, Slug, and Twist. The Snail family of zinc-finger transcriptional repressors directly represses E-cadherin in vitro and in vivo via an interaction between their $\mathrm{COOH}$-terminal region and the $5^{\prime}$-CACCTG-3' sequence in the E-cadherin promoter [7-9]. Snail is reportedly important in several carcinomas, including non-small cell lung carcinomas, ovarian carcinomas, urothelial carcinomas, and hepatocellular carcinoma [10-13]. Studies have also used immunohistochemical analyses to show the clinical significance of Snail overexpression in gastric adenocarcinoma (GC) $[14,15]$. However, few reports on

\section{Biomed Central}


the roles of Snail in GC have included clinicopathological, prognostic, and functional in vitro analyses as well as gene expression results. We therefore evaluated Snail's effect on invasiveness/migratory ability in gastric cancer cell lines, and also investigated the possibility of Snail being used as a predictive marker for evaluating poor prognosis or tumor aggressiveness in GC patients. We also evaluated the gene expression pattern in $45 \mathrm{GC}$ tissues with Snail overexpression, using cDNA microarrays.

\section{Methods}

shRNA lentivirus-mediated silencing and overexpression of Snail in gastric cancer cells

Human gastric cancer cell lines SNU216 and SNU484 were obtained from Korean Cell Line Bank (KCLB) and were authenticated by DNA profiling. These cells cultured in RPMI1640 medium with $10 \%$ fetal bovine serum (FBS), $100 \mathrm{U} / \mathrm{ml}$ penicillin, and $100 \mu \mathrm{g} / \mathrm{ml}$ streptomycin (hyClone, Ogden, UT). All cells were maintained at $37^{\circ} \mathrm{C}$ in 5\% $\mathrm{CO}_{2}$. Lentiviral-based RNA knockdown and overexpression were used for silencing and overexpression of Snail. Lentiviruses expressing either non-target or Snailtargeted shRNAs were used for silencing; a PLKO lentiviral vector targeting Snail or an empty PLKO vector were used for overexpression of Snail in the SNU216 and SNU484 cells. Lentivirus stocks were produced using the Virapower $^{\mathrm{TM}}$ lentiviral packaging mix using the 293FT cell line according to the manufacturer's protocol (Invitrogen, Carlsbad, CA). SNU216 and SNU484 cells grown to 50\% confluence were incubated for $24 \mathrm{~h}$ in a 1:1 dilution of virus:media with $5 \mu \mathrm{g} / \mathrm{ml}$ Polybrene. After a 24-h recovery period in complete media without virus, polyclonal stable cell lines were selected and maintained in media containing $5 \mu \mathrm{g} / \mathrm{ml}$ puromycin. Silencing or overexpression of Snail was determined by RT-PCR and western blotting.

\section{Real time RT-PCR analysis of VEGF, MMP11, and Snail in gastric cancer cells}

Total cellular RNA was extracted using the TRIzol method (Sigma-Aldrich, St Louis, MO, USA). For RT-PCR analysis, 2- $\mu \mathrm{g}$ aliquots of RNA were subjected to cDNA synthesis with $200 \mathrm{U}$ of MMLV reverse transcriptase and $0.5 \mu \mathrm{g}$ of oligo(dT)-15 primer (Promega, Madison, WI, USA). Quantitative real-time PCR was performed with the Rotor-Gene $^{\mathrm{TM}}$ System (QIAGEN, Hilden, Germany) using AccuPower $2 \times$ Greenstar qPCR Master Mix (Bioneer, Daejeon, Korea). cDNA in $1 \mu \mathrm{l}$ of the reaction mixture was amplified with $0.5 \mathrm{U}$ of GoTaq DNA polymerase (Promega) and 10 pmol each of the following sense and antisense primers: GAPDH $5^{\prime}$-TCCATGACAACTTTGGTAT CG-3', 5'-TGTAGCCAAATTCGTTGTCA-3'; Snail 5'CTTCCTCTCCATACCTG-3' ${ }^{\prime}$ 5'-CATAGTTAGTCACA CCTCGT-3'; VEGF 5'-TTGCTGCTCTACCTCCACCA-3', 5'-GCACACAGGATGGCTTGAA-3'; MMP11 5'-CTTG
GCTGCTGTTGTGTGCT-3', 5-AGGTATGGAGCGATG TGACG-3'. The thermal cycling profile was: denaturation for $30 \mathrm{~s}$ at $95^{\circ} \mathrm{C}$, annealing for $30 \mathrm{~s}$ at $52^{\circ} \mathrm{C}$ (depending on the primers used), and extension for $30 \mathrm{~s}$ at $72^{\circ} \mathrm{C}$. For semi-quantitative assessment of expression levels, 30 cycles were used for each PCR reaction. PCR products were sizefractionated on $1.0 \%$ ethidium bromide/agarose gels and quantified under UV transillumination. The threshold cycle (CT) is defined as the fractional cycle number at which the fluorescence passes a fixed threshold above baseline. Relative gene expression was quantified using the average $\mathrm{CT}$ value for each triplicate sample minus the average triplicate CT value for GAPDH. Differences between the control (empty vector) and experiment groups (infected with the lentivirus) were calculated using the formula $2^{-\left([\triangle \mathrm{CT} \text { Lenti }]-\left[{ }^{\Delta} \mathrm{CT} \text { control }\right]\right)}$ and expressed as a fold change in expression according to the comparative threshold cycle method $\left(2{ }^{\Delta \Delta \mathrm{CT}}\right)$ [16].

\section{Western blotting}

Cells were harvested and disrupted in lysis buffer (1\% Triton X-100, 1mM EGTA, 1mM EDTA, 10mM Tris- $\mathrm{HCl}, \mathrm{pH} 7.4$ and protease inhibitors). Cell debris was removed by centrifugation at $10,000 \times g$ for 10 $\min$ at $4{ }^{\circ} \mathrm{C}$. The resulting supernatants were resolved on a $12 \%$ SDS-PAGE under denatured reducing conditions and transferred to nitrocellulose membranes. The membranes were blocked with $5 \%$ non-fat dried milk at room temperature for $30 \mathrm{~min}$ and incubated with primary antibodies. The membranes were washed and incubated with horseradish peroxidaseconjugated secondary antibody. The signal was visualized using an enhanced chemiluminescence (Amersham, Buckinghamshire, UK).

\section{Cell migration and Matrigel invasion assay}

Gastric cancer cells were harvested with $0.05 \%$ trypsin containing $0.02 \%$ EDTA (Sigma-Aldrich), and suspended in RPMI at a concentration of $3 \times 10^{3}$ cells/well. Membrane filters (pore size: $8 \mu \mathrm{m}$ ) in disposable 96-well chemotaxis chambers (Neuro Probe, Gaithersburg, MD) were pre-coated for $4 \mathrm{~h}$ with $5 \mathrm{mg} / \mathrm{ml}$ fibronectin at room temperature. Aliquots $(50 \mu \mathrm{l} /$ well $)$ of the cell suspension were loaded into the upper chambers, and $1 \%$ FBS was loaded into the lower chamber. After 24-h incubation, non-migrating cells were removed from the upper chamber with a cotton swab; cells present on the lower surface of the insert were stained with Hoechst33342 (Sigma-Aldrich). Invasive cells were counted under a fluorescence microscope at $\times 10$ magnification.

For the Matrigel invasion assay, $3 \times 104$ cells/well were seeded in the upper chamber, which was coated with Matrigel $(5 \mathrm{mg} / \mathrm{ml}$ in cold medium, BD Transduction Laboratories, Franklin Lakes, NJ, USA), and serum-free medium containing 1\% FBS or control vehicle was added 
to the lower chamber. After 24-h incubation, nonmigrating cells were removed from the upper chamber with a cotton swab, and cells present on the lower surface of the insert were stained with Hoechst33342 (Sigma-Aldrich). Invasive cells were then counted under a fluorescence microscope at $\times 10$ magnification.

\section{Tissue microarrays, immunohistochemistry, and interpretation of results}

A semi-automated tissue arrayer (Beecher Instruments, WI, USA) was used to construct the tissue microarrays. We obtained 3 tissue cores, each $0.6 \mathrm{~mm}$ in diameter, from tumor blocks taken from GC patients. Cores were not collected from the more invasive frontal or central areas of the tumors. Slides were baked at $60^{\circ} \mathrm{C}$ for 30 min, deparaffinized with xylene, and then rehydrated. The sections were subsequently submerged in citrate antigen retrieval buffer, microwaved for antigen retrieval, treated with $3 \%$ hydrogen peroxide in methanol to quench endogenous peroxidase activity, and then incubated with $1 \%$ bovine serum albumin to block nonspecific binding. Thereafter, the sections were incubated with rabbit anti-Snail (Abcam, UK) overnight at $4^{\circ} \mathrm{C}$. Normal rabbit serum was used as a negative control. After washing, tissue sections were treated with secondary antibody, counterstained with hematoxylin, dehydrated, and mounted. At least 500 tumor cells were counted. The percentage of cells with Snail ${ }^{+}$nuclei was expressed relative to the total number of tumor cells counted. Nuclear expression of Snail was graded by classifying the extent of positive nuclear staining as $\leq 50 \%$, $50-75 \%$, or $\geq 75 \%$.

\section{Clinicopathological and survival analysis of gastric cancer patients}

We studied a cohort of 314 GC patients who each underwent a gastrostomy with lymph node dissection at Pusan National University Hospital (PNUH) between 2005 and 2007. The group comprised 218 men and 96 women with a mean age of 58.3 years (range, 25-83 years). Standard formalin-fixed and paraffin-embedded sections were obtained from the Department of Pathology, PNUH, and the National Biobank of Korea, PNUH. The study was approved by the Institutional Review Board. None of the patients received preoperative radiotherapy and/or chemotherapy. Adjuvant chemotherapy based on 5-FU was administered on patients with stages II, III and IV after curative resection. We assessed several clinicopathological factors according to the Korean Standardized Pathology Report for Gastric Cancer, the Japanese Classification of Gastric Carcinoma ( $3^{\text {rd }}$ English edition), and the American Joint Committee on Cancer Staging Manual ( $7^{\text {th }}$ edition), including tumor site, gross appearance and size, depth of invasion, histological classification (i.e., intestinal or diffuse), and lymphovascular invasion [17-19]. Clinical outcome for each patient was followed from the date of surgery to the date of death or March 1, 2012. Follow-up periods ranged from approximately 1 to 81.5 months (average, 51.4 months). Cases lost to follow-up or death from any cause other than gastric cancer were censored from the survival rate analysis. Clinicopathological features were analyzed using Student's $t$-test, the $\chi^{2}$ test, or Fisher's exact test to test for differences in Snail expression. $\mathrm{Cu}$ mulative survival plots were obtained using the KaplanMeier method, and significance was compared using the log-rank test. Prognostic factors were identified using the Cox regression stepwise method (proportional hazard model), adjusted for the patients' age, gender, tumor site, morphologic type (intestinal versus diffuse). Statistical significance was set at $P<0.05$. Statistical calculations were performed with SPSS version 10.0 for Windows (SPSS Inc., Chicago, IL, USA).

\section{cDNA microarray analysis of GC tissues based on Snail overexpression}

A total of 45 fresh GC tissues were obtained from the National Biobank of Korea, PNUH, and CNUH; approval was obtained from their institutional review boards. Total RNA was extracted from the fresh-frozen tissues using a mirVana RNA Isolation kit (Ambion Inc., Austin, TX). Five hundred nanograms of total RNA was used for cDNA synthesis, followed by an amplification/labeling step (in vitro transcription) using the Illumina TotalPrep RNA Amplification kit (Ambion) to synthesize biotinlabeled cRNA. cRNA concentrations were measured by the RiboGreen method (Quant-iT RiboGreen RNA assay kit; Invitrogen-Molecular Probes, ON, Canada) using a Victor3 spectrophotometer (PerkinElmer, CT), and cRNA quality was determined on a $1 \%$ agarose gel. Labeled, amplified material (1500 ng per array) was hybridized to Illumina HumanHT-12 BeadChips v4.0, according to manufacturer's instructions (Illumina, San Diego, CA). Array signals were developed by streptavidin-Cy3. Arrays were scanned with an Illumina iScan system. The microarray data were normalized using the quantile normalization method in Illumina BeadStudio software. The expression level of each gene was transformed into a $\log ^{2}$ base before further analysis. Excel was primarily used for statistical analyses. Gene expression differences were considered statistically significant if $P<$ 0.05 ; all tests were 2-tailed. Cluster analyses were performed using Cluster and Treeview [20]. The gene ontology (GO) program (http://david.abcc.ncifcrf.gov/) was used to categorize genes into subgroups based on biological function. Fisher's exact test was used to determine whether the proportions of genes in each category differed by group. GC tissues were further 


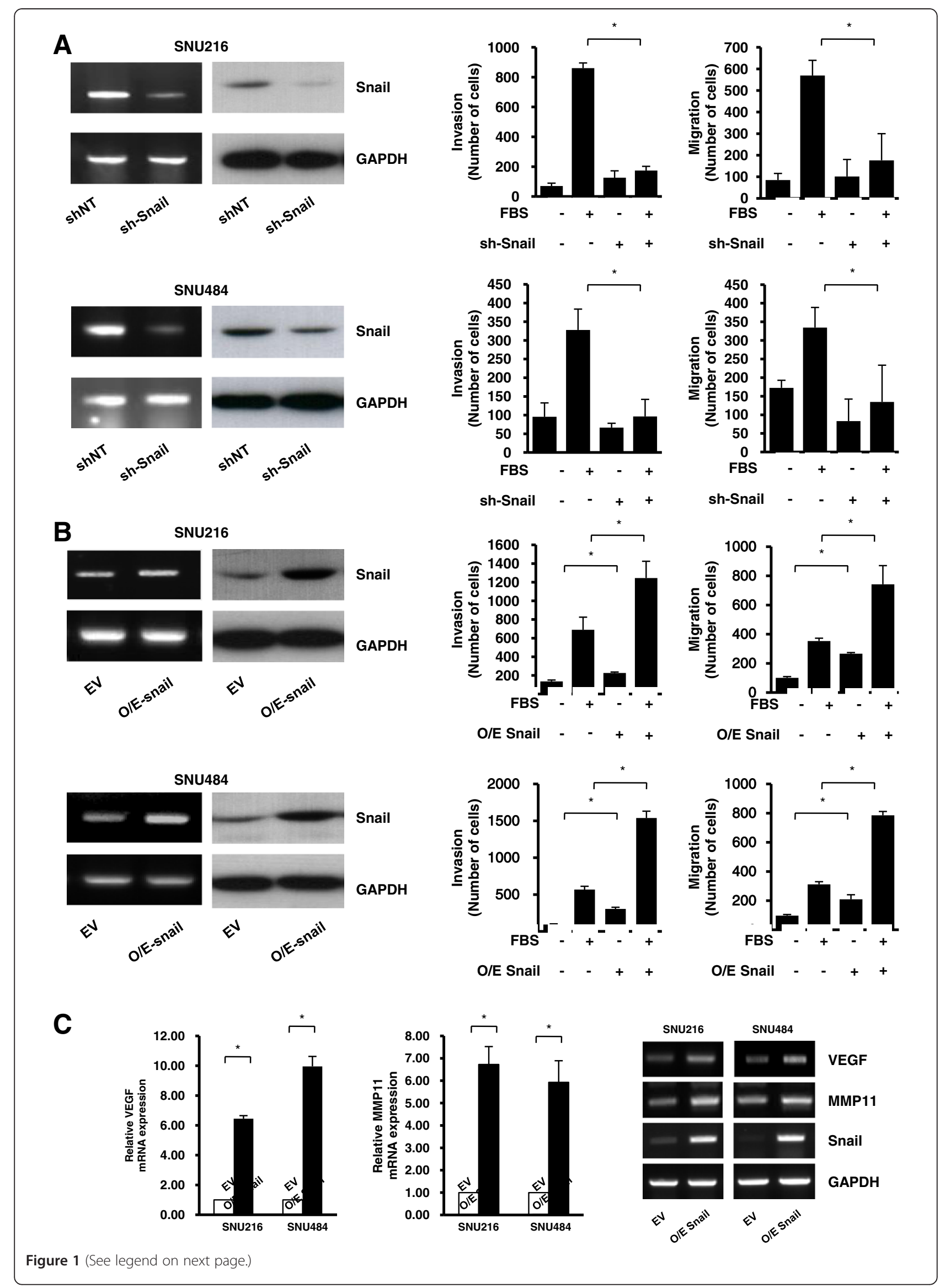


(See figure on previous page.)

Figure 1 Role of Snail in invasion and migration of gastric cancer cell lines. A. SNU216 (upper panel) and SNU484 (lower panel) cells were infected with lentiviruses expressing either non-target shRNA (shNT) or Snail shRNA on day 0, and then harvested on day 7 post-infection. Snail knockdown was determined by RT-PCR and western blotting; stable cell lines were generated for each of the cell lines (sh-Snail). Silencing of Snail in SNU216 and SNU484 cells induced decreased migration and invasion. B. SNU216 (upper panel) and SNU484 (lower panel) cells were infected with lentiviruses expressing either a lentiviral PLKO vector targeting Snail or an empty PLKO vector (EV) on day 0, and then harvested on day 7 post-infection. The overexpression of Snail was determined by RT-PCR and western blotting; stable cell line was generated for each of the cell lines (O/E-snail). Snail overexpression in SNU216 and SNU484 cells induced increased migration and invasion. C. Snail overexpression induced increased mRNA expression of VEGF and MMP11 in SNU216 and SNU484 cells in real-time RT-PCR analysis. Lower panel indicates representative RT-PCR figures for VEGF, MMP11, Snail, and GAPDH. Data show the mean \pm SE of at least 3 independent experiments. ${ }^{*}$ indicates $P<0.05$ by Student's t-test.

divided into those with higher $(\geq 75 \%)$ and lower $(<75 \%)$ levels of Snail expression; differential gene expression between the groups was identified. Primary microarray data are available in NCBI's GEO (Gene Expression Omnibus) database (http://www.ncbi.nlm.nih.gov/geo/ query/acc.cgi?acc=GSE38024).

\section{Results}

\section{Regulation of migration and invasion of gastric cancer} cells by Snail

Lentiviral-based RNA knockdown and overexpression approaches were used to determine Snail's role in invasion and migration of gastric cancer cell lines. SNU216 and
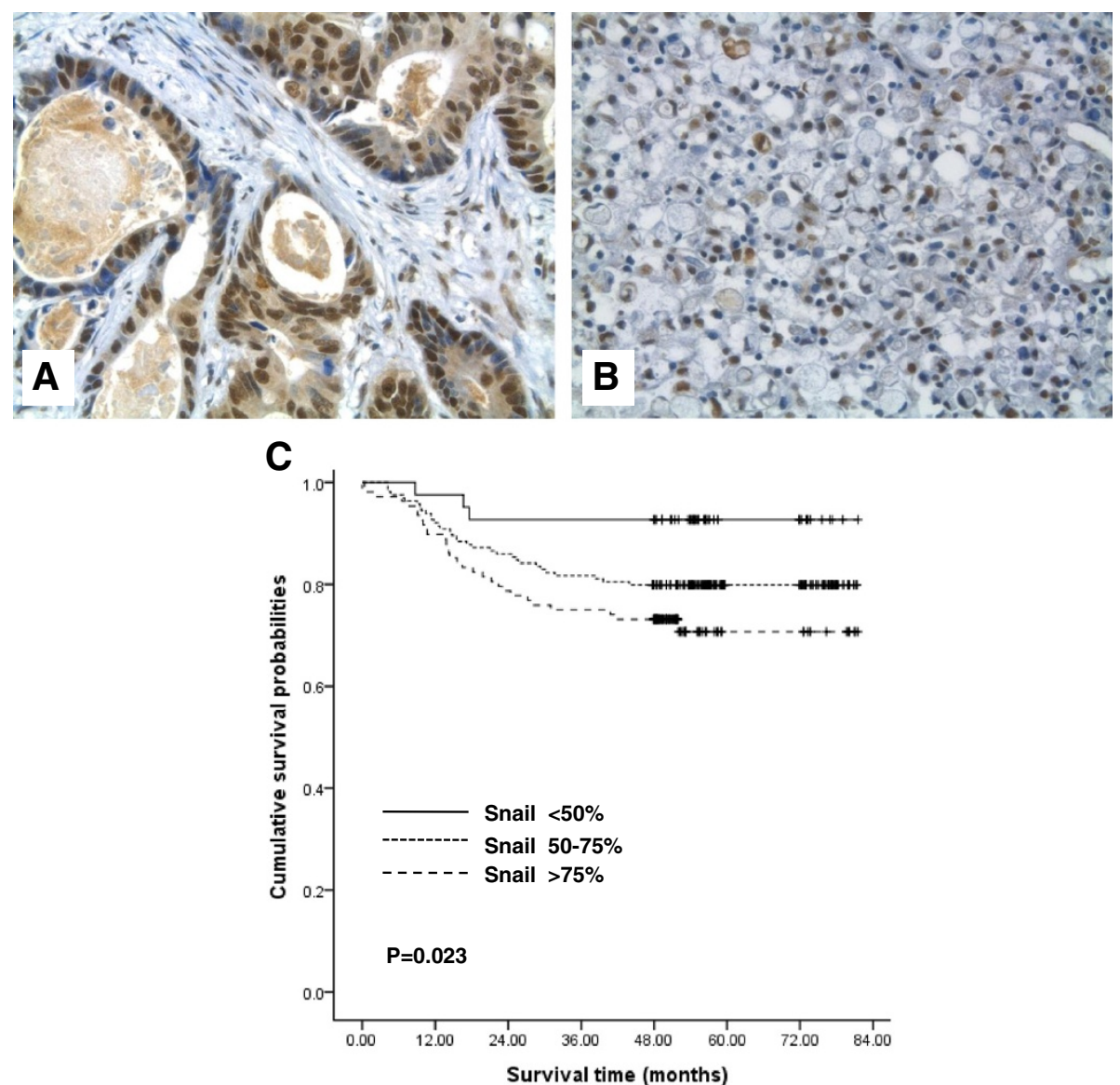

Figure 2 Snail expression in gastric adenocarcinoma (GC) tissue samples and Kaplan-Meir plots of overall survival of 314 GC patients. Snail was mostly expressed in nuclei of GC cells (intestinal type (A), and diffuse type (signet ring) cells (B)) included in tissue array specimens. Some reactive fibroblasts also showed Snail nuclear expression (magnification: $\times 400$ ). C. Kaplan-Meier analysis of overall survival of GC patients based on Snail expression. A linear relationship between increased Snail nuclear expression and shorter survival was seen among GC patients $(P=0.023)$. Log-rank test was used to calculate $P$ values. 
Table 1 Relationship between Snail expression and clinicopathological characteristics in $\mathbf{3 1 4}$ patients with gastric cancer

\begin{tabular}{|c|c|c|c|c|}
\hline & Number of & Snail Posit & & $P$ value \\
\hline & $\begin{array}{l}\text { patients } \\
(\mathrm{N}=314)\end{array}$ & $<75 \%$ & $\geq 75 \%$ & \\
\hline Age (years) & & $58.5 \pm 10.6$ & $59.1 \pm 11.9$ & 0.695 \\
\hline Sex & & & & \\
\hline Male & 218 & 143 & 75 & 0.996 \\
\hline Female & 96 & 63 & 33 & \\
\hline Tumor size & & & & \\
\hline$\leq 4.0 \mathrm{~cm}$ & 192 & 135 & 57 & 0.028 \\
\hline$>4.0 \mathrm{~cm}$ & 122 & 71 & 51 & \\
\hline Location & & & & \\
\hline Upper/Middle & 167 & 112 & 55 & 0.561 \\
\hline Lower & 147 & 94 & 53 & \\
\hline Invasion depth & & & & \\
\hline $\mathrm{T} 1$ & 160 & 127 & 33 & $<0.001$ \\
\hline $\mathrm{T} 2$ & 41 & 26 & 15 & \\
\hline $\mathrm{T} 3$ & 68 & 33 & 35 & \\
\hline T4 & 43 & 19 & 24 & \\
\hline Gross type & & & & \\
\hline Elevated & 77 & 51 & 26 & $<0.001$ \\
\hline Flat/depressed & 131 & 105 & 26 & \\
\hline Excavated & 106 & 50 & 56 & \\
\hline Histological type & & & & \\
\hline Intestinal & 182 & 123 & 59 & 0.609 \\
\hline Diffuse & 122 & 76 & 46 & \\
\hline Mixed & 10 & 7 & 3 & \\
\hline Perineural invasio & & & & \\
\hline Negative & 202 & 150 & 52 & $<0.001$ \\
\hline Positive & 111 & 55 & 56 & \\
\hline Lymphovascular & mboli & & & \\
\hline Negative & 193 & 139 & 54 & 0.002 \\
\hline Positive & 120 & 66 & 54 & \\
\hline Lymph node met & astasis & & & \\
\hline No, N1 & 270 & 186 & 84 & 0.002 \\
\hline $\mathrm{N} 2, \mathrm{~N} 3$ & 44 & 20 & 24 & \\
\hline
\end{tabular}

SNU484 cells used in this study are established gastric adenocarcinoma cell lines from Korean patients. These cells were infected with a lentivirus expressing either nontarget or Snail-targeted shRNAs for silencing. A PLKO lentiviral vector that targeted Snail and an empty PLKO vector were used to induce Snail overexpression in SNU216 and SNU484 cells. Polyclonal stable cell lines were selected using puromycin. Snail expression was determined by RT-PCR and western blotting; stable Snail knockdown (sh-Snail) and Snail overexpression cell lines (OE-Snail) were obtained (Figure 1).

To determine Snail's roles in gastric cancer cell invasion, we measured chemotactic invasion by the cells using the Transwell system with filters pre-coated with Matrigel. To measure migration of gastric cancer cells, we assayed cell migration using a Boyden chamber apparatus. Silencing of Snail by shRNA induced decreased migration and invasion of SNU216 and SNU484 cells, as shown in Figure 1A. In contrast to the Snail silencing results, overexpression of Snail induced increased migration and invasion of SNU216 and SNU484 cells, as shown in Figure 1B. Overexpression of Snail was also associated with increased VEGF and MMP11 (Figure 1C).

\section{Effect of Snail overexpression on tumor aggressiveness and GC patient survival}

Positive nuclear staining for Snail at levels of $\leq 50 \%, 50$ $75 \%$, and $\geq 75 \%$ was observed in $13.4 \%$ (42/314), $52.2 \%$ (164/314), and 34.4\% (108/314), respectively, of the 314 GC patients in immunohistochemical analysis. Snail expression was noted in intestinal and diffuse type of GCs (Figure 2A, B). Snail overexpression ( $\geq 75 \%$ positivity) significantly correlated with tumor size, gross type, depth of invasion, lymphovascular invasion, perineural invasion, and lymph node metastasis (Table 1). Snail overexpression was also associated with increased tumor size $(P=0.028)$ and excavated gross type $(P<0.001)$; and increased tumor invasiveness, i. e., higher $\mathrm{T}$ stage $(P<0.001)$ and the presence of perineural invasion $(P<0.001)$ and lymphovascular tumor emboli $(P=0.002)$. Increased lymph node metastasis was also related to Snail overexpression $(P=0.002)$.In accordance with the above data showing the positive relationship between Snail overexpression and GC aggressiveness, Snail overexpression significantly correlated with overall survival

Table 2 Multivariate survival analysis with Cox regression model in 314 gastric cancers

\begin{tabular}{lllll}
\hline Variables & B & SE & HR (95\% Cl) & P \\
\hline Age $(\leq 59$ versus $>59)$ & -0.438 & 0.264 & $0.645(0.385-1.082)$ & 0.097 \\
Gender (male versus female) & -0.037 & 0.267 & $0.963(0.571-1.626)$ & 0.889 \\
Site (upper and middle versus lower) & 0.635 & 0.264 & $1.887(1.126-3.164)$ & 0.016 \\
Lauren (intestinal vs diffuse) & -0.537 & 0.263 & $0.585(0.349-0.978)$ & 0.041 \\
Snail $(\geq 75 \%$ versus $<75 \%)$ & -0.528 & 0.248 & $0.590(0.363-0.958)$ & 0.033 \\
\hline
\end{tabular}

Note: B, coefficient; $\mathrm{HR}$, hazard ratio; $\mathrm{Cl}$, confidence interval. 
Table 3 Genes differentially expressed in GC specimens with higher levels of Snail expression

\begin{tabular}{|c|c|c|}
\hline PROBE_ID & SYMBOL & NAME \\
\hline \multicolumn{3}{|c|}{ Genes upregulated in specimens with higher levels $(\geq 75 \%)$ of Snail expression $(P<0.05)$} \\
\hline ILMN_2374449 & SPP1 & Secreted phosphoprotein 1 \\
\hline ILMN_2337923 & TPD52L1 & Tumor protein D52-like 1 \\
\hline ILMN_1679838 & WBP5 & WW domain binding protein 5 \\
\hline ILMN_2078592 & C6orf105 & Androgen-dependent TFPI-regulating protein \\
\hline ILMN_1714383 & TPD52L1 & Tumor protein D52-like 1 \\
\hline ILMN_1674817 & Clorf115 & Chromosome 1 open reading frame 115 \\
\hline ILMN_1813561 & SCIN & Scinderin \\
\hline ILMN_1759818 & SORL 1 & Sortilin-related receptor, L(DLR class) A repeats containing \\
\hline ILMN_1745686 & MFHAS1 & Malignant fibrous histiocytoma amplified sequence 1 \\
\hline ILMN_2060115 & SORL1 & Sortilin-related receptor, L(DLR class) A repeats containing \\
\hline ILMN_2337263 & PKIB & Protein kinase (CAMP-dependent, catalytic) inhibitor beta \\
\hline ILMN_2173835 & FTHL3 & Ferritin, heavy polypeptide 1 pseudogene 3 \\
\hline ILMN_1791057 & IFNAR2 & Interferon (alpha, beta and omega) receptor 2 \\
\hline ILMN_1807114 & LOC255620 & Similar to unc-93 homolog B1 (C. elegans), transcript variant 1 (LOC255620), mRNA \\
\hline ILMN_1669393 & GGT1 & Gamma-glutamyltransferase 1 \\
\hline ILMN_1685798 & MAGEA6 & Melanoma antigen family A, 6 \\
\hline ILMN_3269395 & GGT2 & Gamma-glutamyltransferase 2 \\
\hline ILMN_1669833 & $\mathrm{SH} 2 \mathrm{~B} 2$ & SH2B adaptor protein 2 \\
\hline ILMN_3238534 & LOC100133817 & Hypothetical protein LOC100133817 \\
\hline ILMN_2099315 & TRPM8 & Transient receptor potential cation channel, subfamily M, member 8 \\
\hline ILMN_3298065 & LOC729195 & Similar to apical early endosomal glycoprotein \\
\hline ILMN_1717726 & FLJ43752 & Long intergenic non-protein coding RNA 336 \\
\hline ILMN_1670452 & ANKRD20A1 & Ankyrin repeat domain 20 family, member $\mathrm{A} 1$ \\
\hline ILMN_3201060 & LOC100132655 & Hypothetical protein LOC100132655 \\
\hline ILMN_3282829 & LOC727913 & Similar to iduronate 2-sulfatase (Hunter syndrome) \\
\hline ILMN_2339691 & SYVN1 & Synovial apoptosis inhibitor 1, synoviolin \\
\hline ILMN_1785549 & SLC30A2 & Solute carrier family 30 (zinc transporter), member 2 \\
\hline ILMN_3191898 & LOC100129630 & Hypothetical LOC100129630 \\
\hline ILMN_1704204 & LOC642204 & Ankyrin repeat domain-containing protein 26-like \\
\hline ILMN_1682280 & LOC647753 & Hypothetical protein LOC647753 \\
\hline ILMN_3201944 & LOC646438 & Hypothetical LOC646438 \\
\hline ILMN_2233314 & SPANXA1 & Sperm protein associated with the nucleus, $X$-linked, family member A1 \\
\hline ILMN_3305980 & NS3BP & NS3BP \\
\hline ILMN_1747850 & CRIM2 & Kielin/chordin-like protein \\
\hline ILMN_1700590 & LOC645590 & Similar to CAMP-dependent protein kinase type I-beta regulatory subunit \\
\hline ILMN_1766316 & FLJ32679 & Golgin-like hypothetical protein LOC440321 \\
\hline ILMN_1890741 & Hs.552561 & Pancreatic islet cDNA clone hbt09690 3, mRNA sequence \\
\hline ILMN_3308255 & MIR33A & MicroRNA 33a \\
\hline ILMN_1815716 & $L M L N$ & Leishmanolysin-like (metallopeptidase M8 family) \\
\hline ILMN_1654945 & DNMT3A & DNA (cytosine-5-)-methyltransferase 3 alpha \\
\hline ILMN_2256050 & SERPINA1 & Serpin peptidase inhibitor, clade A (alpha-1 antiproteinase, antitrypsin), member 1 \\
\hline ILMN_1759487 & EGFLAM & EGF-like, fibronectin type III and laminin G domains \\
\hline ILMN_1760410 & LOC653086 & Similar to RAN-binding protein 2-like 1 isoform 2 \\
\hline
\end{tabular}




\section{Table 3 Genes differentially expressed in GC specimens with higher levels of Snail expression (Continued)}

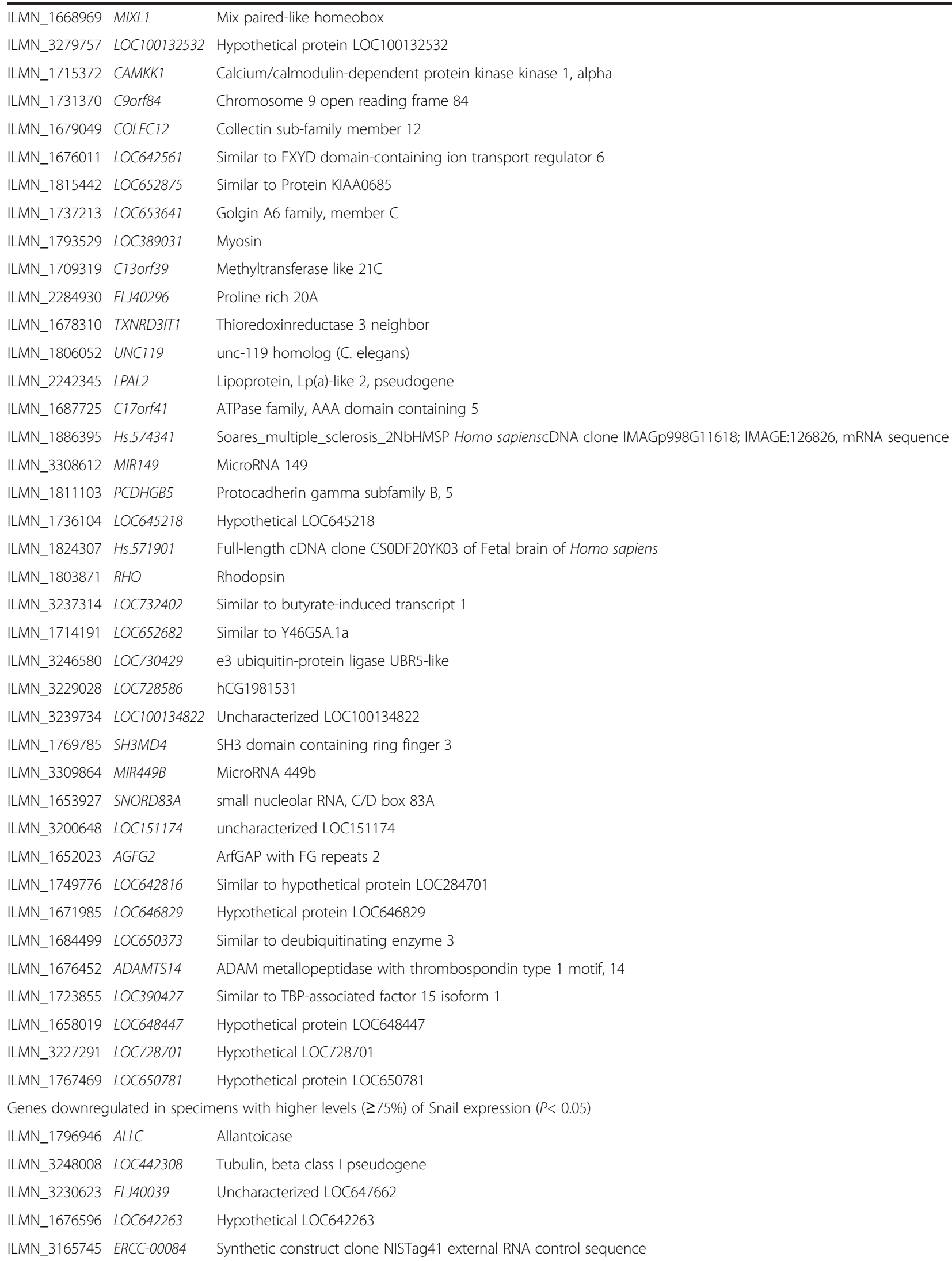




\section{Table 3 Genes differentially expressed in GC specimens with higher levels of Snail expression (Continued)}

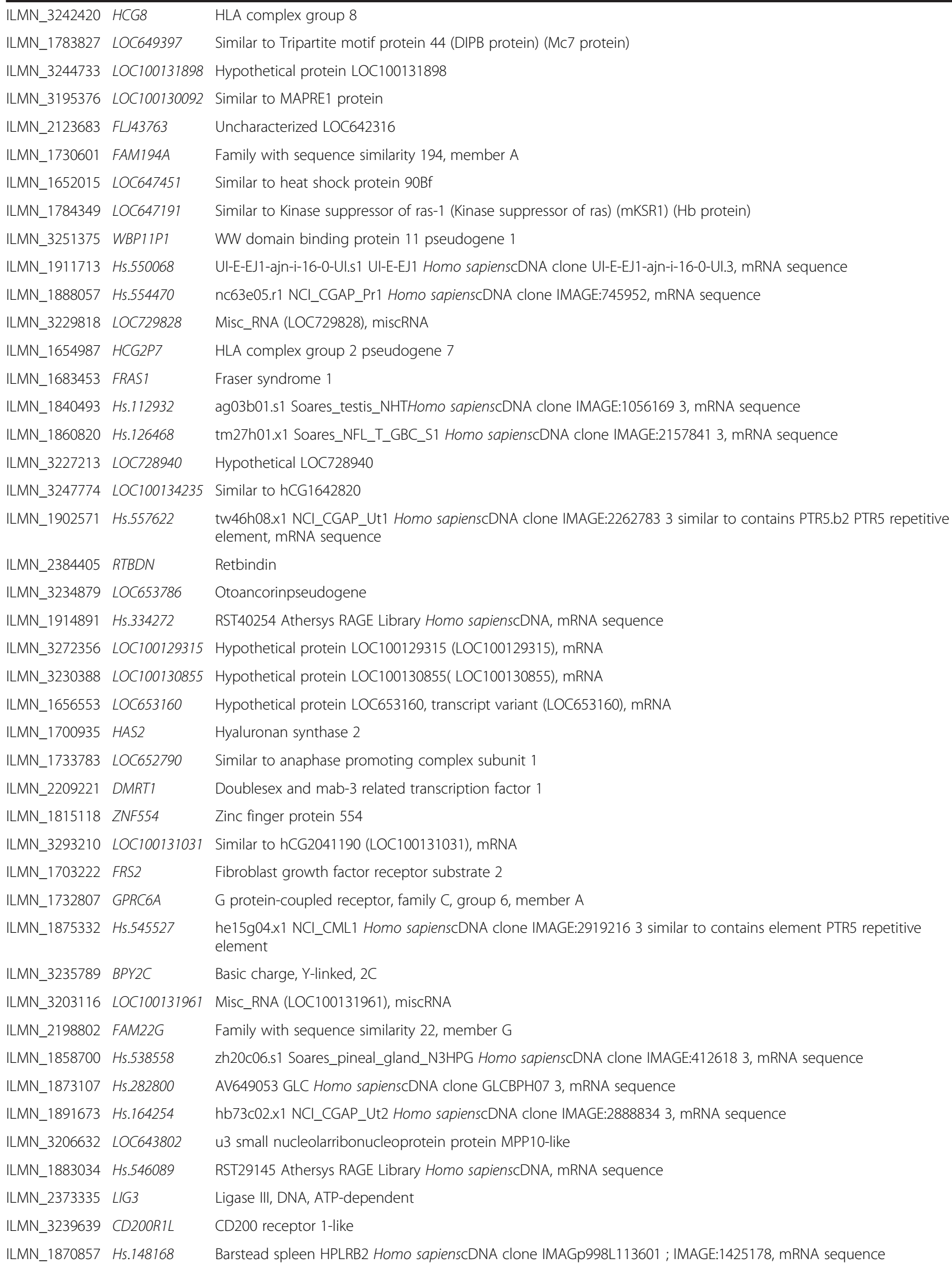




\section{Table 3 Genes differentially expressed in GC specimens with higher levels of Snail expression (Continued)}

\begin{tabular}{|c|c|c|}
\hline ILMN_1813909 & CRSP2 & Mediator complex subunit 14 \\
\hline ILMN_1891885 & Hs.332843 & qg83a07.x1 Soares_NFL-T_GBC_S1 Homo sapienscDNA clone IMAGE:1841748, mRNA sequence \\
\hline ILMN_3235126 & LOC100133558 & Similar to hCG1642170 \\
\hline ILMN_1677186 & MGC52498 & Family with sequence similarity 159 , member A \\
\hline ILMN_3252608 & HCRP1 & Hepatocellular carcinoma-related HCRP1 \\
\hline ILMN_1652871 & PLSCR5 & Phospholipid scramblase family, member 5 \\
\hline ILMN_1698894 & OR5AS1 & Olfactory receptor, family 5 , subfamily AS, member 1 \\
\hline ILMN_1705828 & RICTOR & RPTOR independent companion of MTOR, complex 2 \\
\hline ILMN_1683046 & OR6Y1 & Olfactory receptor, family 6 , subfamily $Y$, member 1 \\
\hline ILMN_2114812 & ONECUT1 & One cut homeobox 1 \\
\hline ILMN_1770248 & PDLIM2 & PDZ and LIM domain 2 (mystique) \\
\hline ILMN_1784272 & CD1E & CD1e molecule \\
\hline ILMN_1755635 & FLJ33534 & Hypothetical protein FLJ33534 (FLJ33534), mRNA \\
\hline ILMN_1799067 & TRY1 & Protease, serine, 1 (trypsin 1) \\
\hline ILMN_1693448 & LOC643811 & Similar to FERM domain containing 6 \\
\hline ILMN_1723323 & HCG4 & HLA complex group 4 (non-protein coding) \\
\hline ILMN_1865604 & $H s .253267$ & 60270330F1 NCl_CGAP_Skn3 Homo sapienscDNA clone IMAGE:4800534 5, mRNA sequence \\
\hline ILMN_3308698 & MIR1276 & MicroRNA 1276 \\
\hline ILMN_1714014 & LOC644491 & NMDA receptor regulated 2 pseudogene \\
\hline ILMN_2114185 & Clorf104 & RUSC1 antisense RNA 1 (non-protein coding) \\
\hline ILMN_1911044 & Hs.540915 & nf66b06.s1 NCl_CGAP_Co3 Homo sapienscDNA clone IMAGE:924851 3, mRNA sequence \\
\hline ILMN_1748543 & STRC & Stereocilin \\
\hline ILMN_1675221 & $D G K Z$ & Diacylglycerol kinase, zeta \\
\hline ILMN_1726263 & LOC653748 & $\begin{array}{l}\text { Similar to dipeptidylaminopeptidase-like protein } 6 \text { (dipeptidylpeptidase VI) (dipeptidylpeptidase 6) (dipeptidyl } \\
\text { peptidase VI-like protein) (dipeptidylaminopeptidase-related protein) (DPPX) }\end{array}$ \\
\hline ILMN_1817113 & Hs.547985 & UI-H-BIOp-abm-h-10-0-UI.s1 NCI_CGAP_Sub2 Homo sapienscDNA clone IMAGE:2712450 3, mRNA sequence \\
\hline ILMN_1793525 & KIR2DS3 & Killer cell immunoglobulin-like receptor, two domains, short cytoplasmic tail, 3 \\
\hline ILMN_2415617 & C10orf72 & V-set and transmembrane domain containing 4 \\
\hline ILMN_1746277 & MLLT4 & Myeloid/lymphoid or mixed-lineage leukemia (trithorax homolog, Drosophila); translocated to, 4 \\
\hline ILMN_1678246 & LOC644001 & Hypothetical protein LOC644001 \\
\hline ILMN_3257856 & LOC100130938 & Hypothetical LOC100130938 (LOC100130938), mRNA \\
\hline ILMN_1865630 & Hs.116333 & Soares_testis_NHTHomo sapienscDNA clone IMAGp998A031828, mRNA sequence \\
\hline ILMN_2152028 & LOC642452 & Hypothetical LOC642452 (LOC642452), mRNA \\
\hline ILMN_3244579 & LOC649330 & Heterogeneous nuclear ribonucleoprotein C-like \\
\hline ILMN_1905832 & Hs.564127 & UI-E-DW1-ahc-g-05-0-Ul.r1 UI-E-DW1 Homo sapienscDNA clone UI-E-DW1-ahc-g-05-0-UI.5, mRNA sequence \\
\hline ILMN_1897251 & Hs.547715 & UI-E-EJO-ahv-e-11-0-UI.s1 UI-E-EJO Homo sapienscDNA clone UI-E-EJO-ahv-e-11-0-UI 3, mRNA sequence \\
\hline ILMN_1782800 & LOC651410 & Hypothetical protein LOC651410 \\
\hline ILMN_1732554 & ZNF346 & Zinc finger protein 346 \\
\hline ILMN_1674014 & LOC653878 & $\begin{array}{l}\text { Similar to Cytosolic acyl coenzyme A thioester hydrolase, inducible (Long chain acyl-CoA thioester hydrolase) (Long } \\
\text { chain acyl-CoA hydrolase) (CTE-I) (CTE-Ib) }\end{array}$ \\
\hline ILMN_1911501 & Hs.543905 & xi89f08.x1 NCl_CGAP_Mel3 Homo sapienscDNA clone IMAGE:265999 3, mRNA sequence \\
\hline ILMN_1878305 & Hs.262789 & xk07d09.x1 NCI_CGAP_Co20 Homo sapienscDNA clone IMAGE:2666033 3, mRNA sequence \\
\hline ILMN_1858245 & Hs.156566 & Soares_testis_NHTHomo sapienscDNA clone IMAGp998M073519, mRNA sequence \\
\hline ILMN_1704313 & GSTCD & Glutathione S-transferase, C-terminal domain containing \\
\hline ILMN_1707398 & ESRRB & Estrogen-related receptor beta \\
\hline ILMN_3307954 & L3MBTL4 & I(3)mbt-like 4 (Drosophila) \\
\hline
\end{tabular}




\section{Table 3 Genes differentially expressed in GC specimens with higher levels of Snail expression (Continued)}

\begin{tabular}{|c|c|c|}
\hline ILMN_1851244 & Hs.59368 & UI_H_Bl1_aex-h-12-0-Ul.s1 NCI_CGAP_Sub3 Homo sapienscDNA clone IMAGE:2720903 3, mRNA \\
\hline ILMN_1828556 & Hs.541581 & nac23e12.x1 Lupski_sciatic_nerveHomo sapienscDNA clone IMAGE:3394270 3, mRNA sequence \\
\hline ILMN_1692894 & LOC654042 & Similar to dehydrogenase/reductase (SDR family) member 4 like 2 \\
\hline ILMN_1893728 & Hs.377660 & Homo sapienscDNA FLJ26242 fis, clone DMC00770 \\
\hline ILMN_1667005 & LOC652676 & Similar to similar to hypothetical protein FLJ36144 \\
\hline ILMN_3241607 & LOC100132106 & Hypothetical LOC100132106 \\
\hline ILMN_1797503 & GOLGA8G & Golgin A8 family, member $\mathrm{G}$ \\
\hline ILMN_1828034 & Hs.154513 & ik89c11.z1 Human insulinomaHomo sapienscDNA clone IMAGE:6027645 3, mRNA sequence \\
\hline ILMN_1886816 & Hs.544491 & qq31a07.x1 Soraes_NhHMPu_S1 Homo sapienscDNA clone IMAGE:1934100 3, mRNA sequence \\
\hline ILMN_1847950 & Hs.505398 & wq87c02.x1 NCl_CGAP_GC6 Homo sapienscDNA clone IMAGE:2479010 3, mRNA sequence \\
\hline ILMN_1734479 & ACCN3 & Acid-sensing (proton-gated) ion channel 3 \\
\hline ILMN_1675025 & H2BFM & H2B histone family, member M \\
\hline ILMN_2073279 & SIM1 & Single-minded homolog 1 (Drosophila) \\
\hline ILMN_1910185 & Hs.98563 & zw57h03.s1 Soares_total_fetus_Nb2HF8_9w Homo sapienscDNA clone IMAGE:774197 3, mRNA sequence \\
\hline ILMN_3251491 & UQCRB & Ubiquinol-cytochrome $\mathrm{c}$ reductase binding protein \\
\hline ILMN_2180315 & ATG4D & ATG4 autophagy related 4 homolog D (S. cerevisiae) \\
\hline ILMN_1885583 & Hs.542934 & Homo sapienscDNA FLJ26431 fis, clone KDN01390 \\
\hline ILMN_1743301 & MSR1 & Macrophage scavenger receptor 1 \\
\hline ILMN_1809820 & LOC648963 & Similar to retinitis pigmentosa 1 -like 1 \\
\hline ILMN_1869348 & Hs.460114 & UI-E-EJ0-ahv-d-07-0-UI.s1 UI-E-EJ0 Homo sapienscDNA clone UI-E-EJ0-ahv-d-07-0-UI 3, mRNA sequence \\
\hline ILMN_1711332 & TFEC & Transcription factor EC \\
\hline ILMN_2228538 & IRAKIBP1 & Interleukin-1 receptor-associated kinase 1 binding protein 1 \\
\hline ILMN_1756455 & IL5RA & Interleukin 5 receptor, alpha \\
\hline ILMN_1719202 & ZNF174 & Zinc finger protein 174 \\
\hline ILMN_1847029 & Hs.553290 & HESC3_84_D06.g1_A036 Human embryonic stem cells Homo sapienscDNA clone IMAGE:7483454 5, mRNA sequence \\
\hline ILMN_1740217 & HACE1 & HECT domain and ankyrin repeat containing E3 ubiquitin protein ligase 1 \\
\hline ILMN_1787464 & LOC651296 & Similar to RAB, member of RAS oncogene family-like $2 B$ isoform 1 \\
\hline ILMN_1734096 & DCLRETA & DNA cross-link repair $1 \mathrm{~A}$ \\
\hline ILMN_2391333 & CYP20A1 & Cytochrome P450, family 20, subfamily A, polypeptide 1 \\
\hline ILMN_2226314 & DBR1 & Debranching enzyme homolog 1 (S. cerevisiae) \\
\hline ILMN_2379560 & CDC14B & CDC14 cell division cycle 14 homolog B (S. cerevisiae) \\
\hline ILMN_2078466 & DZIP1L & DAZ interacting protein 1-like \\
\hline ILMN_1653039 & LOC642934 & Hypothetical protein LOC642934 (LOC642934), mRNA \\
\hline ILMN_2044293 & KBTBD7 & Kelch repeat and BTB (POZ) domain containing 7 \\
\hline ILMN_1809951 & ZNF200 & Zinc finger protein 200 \\
\hline ILMN_1760280 & NXT1 & NTF2-like export factor 1 \\
\hline ILMN_1657796 & STMN1 & Stathmin 1 \\
\hline ILMN_1793578 & ZFP37 & Zinc finger protein 37 homolog (mouse) \\
\hline
\end{tabular}

among GC patients $(P=0.023)$ (Figure $2 \mathrm{C})$. A linear relationship was observed between increased nuclear expression of Snail and shortened survival ( $\leq 50 \%$ : $76.6 \pm 2.7$ months; 50-75\%: $68.5 \pm 2.0$ months; $\geq 75 \%$ : $63.3 \pm 2.8$ months). Snail overexpression ( $\geq 75 \%$ positivity) was identified as an independent predictor of poor prognosis in 314 patients with GC, adjusted for age, sex, histologic classification, and tumor location, using a Cox regression proportional hazard model $(P=0.033$; Table 2$)$.

\section{Identification of gene expression patterns based on Snail} overexpression using cDNA microarrays

cDNA microarrays were used to compare gene expression profiles of 45 GC specimens. We identified 213 


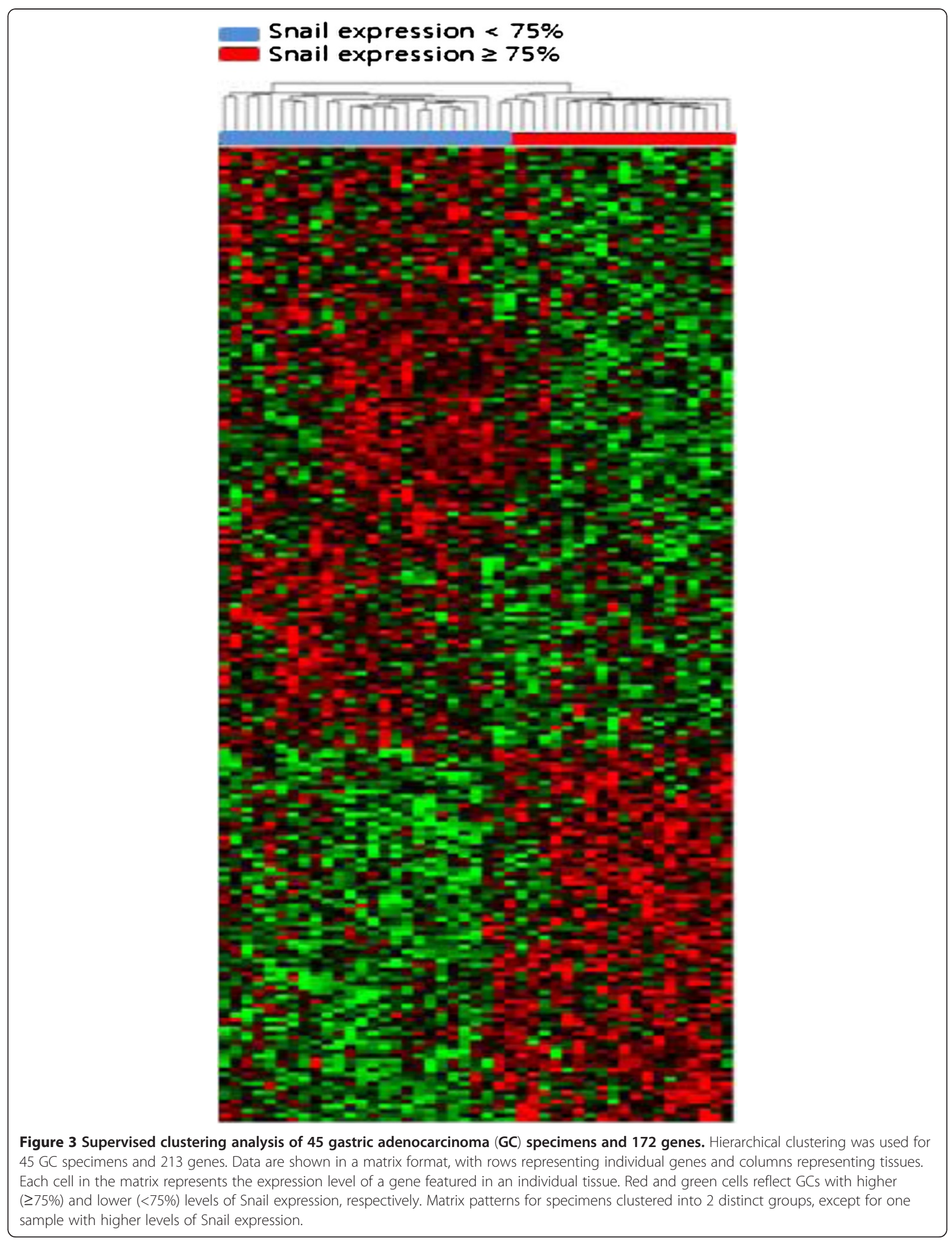


genes that were differentially expressed at significant levels $(P<0.05)$ between GC specimens with higher $(\geq 75 \%)$ and lower levels $(<75 \%)$ of Snail expression (Table 3). Of these 213 genes, 82 were upregulated and 131 were downregulated in the GC specimens with higher levels $(\geq 75 \%)$ of Snail expression. We used hierarchical clustering analysis to assess the 213 genes and 45 GC specimens; supervised clustering analysis gave patterns for samples with higher and lower levels of Snail expression clustered into 2 distinct groups, except for one sample with higher levels of Snail expression (Figure 3). To investigate the biological functions involved in discriminating genes, we performed a GO category analysis. Eleven genes were associated with regulating cancer cell-ECM adhesion $(P<0.021)$ and ECM protein regulation $(P<0.028$, Table 4$)$. Most have been implicated in cancer. ONECUT1, ADAMTS, IFNAR2, MSR1, and SORL1 affect migration or metastasis, a process that involves attachment of tumor cells to the basement membrane, degradation of local connective tissue, and penetration and migration of tumor cells through stroma [21-25].

\section{Discussion}

Snail is reportedly a key regulator of tumor progression and metastasis via increased MMP expression and tumor invasion $[26,27]$. Similarly, we found that upregulated Snail expression increased gastric cancer cell invasion/migration, whereas downregulated Snail expression decreased gastric cancer cell invasion/migration. Yang et al. reported that Snail overexpression in hepatocellular carcinoma cell lines induced increased invasiveness/metastasis [13]. In addition, Kosaka et al. reported that Snail knockdown was associated with decreased invasive capacity of a urothelial carcinoma cell line, supporting our results [12]. We also found that Snail overexpression induced increased expression of VEGF and MMP11, which are known markers of tumor invasion and metastasis. Jin et al. also reported that Snail knockdown by antisense Snail was associated with inhibited MMP activity, demonstrating the importance of regulating MMP activity in cancer metastasis. ${ }^{10}$ Furthermore, Peinado et al. reported that I MDCK cells with Snail overexpression had increased angiogenesis and VEGF [28]. We also observed increased VEGF in gastric cancer cells with Snail overexpression.

The clinical importance of Snail in various carcinomas, including non-small cell lung carcinomas, ovarian carcinomas, urothelial carcinomas, hepatocellular carcinoma, and breast cancer, is well known, as is the poor prognosis associated with Snail overexpression [10-13,29]. However, only limited immunohistochemical data have been available on Snail expression in GC, with no comprehensive clinical and functional analysis of Snail expression in GC patients. Kim et al. reported immunohistochemical data indicating that Snail expression was an independent indicator of prognosis in tissue microarray specimens [14]. Rye et al. reported that the combination of Snail, vimentin, E-cadherin, and CD44 was also significantly associated with poor prognosis in gastric cancer [15]. In contrast, no

Table 4 Cellular functions of selected genes that are differentially expressed in GC specimens that overexpress Snail

\begin{tabular}{|c|c|c|c|c|}
\hline Probe ID & Gene acronym & Gene name & Accession No. & $P$ value \\
\hline \multicolumn{5}{|c|}{ Cancer cell-ECM adhesion } \\
\hline ILMN_1759487 & EGFLAM & EGF-like, fibronectin type III, and laminin G domains ( $\uparrow$ ) & NM_182801 & 0.005 \\
\hline ILMN_2114812 & ONECUT1 & One cut homeobox $1(\downarrow)$ & NM_004498 & 0.002 \\
\hline ILMN_2374449 & SPP1 & Secreted phosphoprotein $1(\uparrow)$ & NM_000582 & 0.004 \\
\hline \multicolumn{5}{|c|}{ ECM protein regulation } \\
\hline ILMN_1676452 & ADAMTS14 & ADAM metallopeptidase with thrombospondin type 1 motif, $14(\uparrow)$ & NM_080722 & 0.005 \\
\hline ILMN_1759487 & EGFLAM & EGF-like, fibronectin type III, and laminin G domains ( $\uparrow$ ) & NM_182801 & 0.005 \\
\hline ILMN_1683453 & FRAS1 & Fraser syndrome $1(\downarrow)$ & NM_020875 & 0.003 \\
\hline ILMN_1791057 & IFNAR2 & Interferon (alpha, beta, and omega) receptor $2(\uparrow)$ & NM_207585 & 0.001 \\
\hline ILMN_1756455 & ILSRA & Interleukin 5 receptor, alpha $(\downarrow)$ & NM_000564 & 0.004 \\
\hline ILMN_1747850 & CRIM2 & Kielin/chordin-like protein $(\uparrow)$ & NM_199349 & 0.005 \\
\hline ILMN_1743301 & MSR1 & macrophage scavenger receptor $1(\downarrow)$ & NM_002445 & 0.002 \\
\hline ILMN_2374449 & SPP1 & secreted phosphoprotein $1(\uparrow)$ & NM_000582 & 0.004 \\
\hline ILMN_2256050 & SERPINA1 & $\begin{array}{l}\text { Serpin peptidase inhibitor, clade A (alpha-1 antiproteinase, antitrypsin), } \\
\text { member } 1(\uparrow)\end{array}$ & NM_000295 & 0.002 \\
\hline $\begin{array}{l}\text { ILMN_2060115, } \\
\text { ILMN_1759818 }\end{array}$ & SORL1 & Sortilin-related receptor, L(DLR class) A repeats-containing $(\uparrow)$ & NM_003105 & $\begin{array}{l}0.003 \\
<0.001\end{array}$ \\
\hline
\end{tabular}


significant correlation between tumor stage and Snail expression was noted in upper gastrointestinal tract adenocarcinoma, including cancers of the esophagus, cardia, and stomach [30]. In our study, overexpression of Snail $(\geq 75 \%$ nuclear Snail expression) was significantly associated with tumor progression, lymph node metastases, lymphovascular invasion, perineural invasion, and poor prognosis in GC patients. Recently, He et al. reported Snail to be an independent prognostic predictor of patient survival among gastric cancer patients; this is in agreement with our data [31]. Although 5-FU based adjuvant chemotherapy for advanced or metastatic gastric adenocarcinoma was usually performed in our cohort, further work is required to reveal exact significance of Snail expresssion as predictor of chemotherapy response in gastric adenocarcinoma. For the practical use of Snail as a tissue biomarker in predicting lymph node metastasis and poor prognosis, we defined a cut-off value of $75 \%$ positive nuclear expression for Snail overexpression. There are wide variations in cut-off values for Snail overexpression in different types of cancer; for example, $75 \%$ is used in non-small cell lung carcinoma [11], 100 (score of mean percentage $\times$ intensity, range $0-300$ ) is used in urothelial carcinomas [12], and 50\% is used in hepatocellular carcinoma [13]. For gastric cancers, cut-off values of $10 \%$ [14] and 5\% [15] positive nuclear expression of Snail have been reported. Further work is required to determine a practical consensus cut-off value for Snail overexpression.

A total of 213 genes that were differentially expressed among GC samples with higher $(\geq 75 \%)$ and lower levels of Snail expression were clustered into 2 distinct groups: those associated with regulation of cancer cell-ECM adhesion, and those associated with ECM protein regulation, such as ONECUT1 [21], ADAMTS [22], IFNAR2 [23], MSR1[24], and SORL1 [25]. These functions indicate that Snail greatly affects cancer cell migration and metastasis by regulating attachment of tumor cells to basement membranes, degradation of local connective tissue, and penetration and migration of tumor cells through stroma.

\section{Conclusions}

In this study, we showed that Snail overexpression induced increased migration and invasion in gastric cancer cell lines. Snail overexpression was also significantly associated with tumor progression, lymph node metastases, lymphovascular invasion, perineural invasion, and poor prognosis in GC patients. We identified 213 genes that were differentially expressed in GC tissues that overexpressed Snail, including genes related to metastasis and invasion by tumor cells. Our results indicate that Snail is crucial in controlling progression and metastasis of gastric cancer. Thus Snail may be used as a predictive biomarker for evaluating prognosis or aggressiveness of GCs.

\section{Competing interests}

The authors declare that they have no competing interests.

\section{Authors' contributions}

NRS, EHJ, CIC and DYP were involved in the design of the study, collected the clinical data, performed the immunohistochemical analysis and drafted the manuscript. HJM performed in vitro study. CHK performed the analysis of microarray data and helped to draft the manuscript. ISC provided general support and helped to analyze the microarray data. GHK, TYJ, DHK and JHL provided the study materials or patients. DYP supervised the study. All authors read and approved the final manuscript.

\section{Acknowledgements}

This study was supported by grant 0920050 from the National R\&D Program for Cancer Control, Ministry for Health, Welfare and Family Affairs, Republic of Korea.

\section{Author details}

${ }^{1}$ Department of Pathology, Pusan National University Hospital and Pusan National University School of Medicine, 1-10 Ami-Dong, Seo-Gu, Busan 602-739, South Korea. ${ }^{2}$ Department of Internal Medicine, Pusan National University Hospital and Pusan National University School of Medicine, Busan, South Korea. ${ }^{3}$ Department of Surgery, Pusan National University Hospital and Pusan National University School of Medicine, Busan, South Korea.

${ }^{4}$ BioMedical Research Institute, Pusan National University Hospital, Busan, South Korea. ${ }^{5}$ Department of Pathology, Cheonam National University, Gwangju, South Korea. ${ }^{6}$ Korean Bioinformation Center, Korea Research Institute of Bioscience and Biotechnology, Daejeon, South Korea.

Received: 17 July 2012 Accepted: 12 November 2012

Published: 14 November 2012

\section{References}

1. Polyak K, Weinberg RA: Transitions between epithelial and mesenchymal states: acquisition of malignant and stem cell traits. Nat Rev Cancer 2009, 9:265-273.

2. Thompson EW, Newgreen DF, Tarin D: Carcinoma invasion and metastasis: a role for epithelial-mesenchymal transition? Cancer Res 2005, 65:5991-5995.

3. Thiery JP, Sleeman JP: Complex networks orchestrate epithelialmesenchymal transitions. Nat Rev Mol Cell Biol 2006, 7:131-142.

4. Kang Y, Massagué J: Epithelial-mesenchymal transitions: twist in development and metastasis. Cell 2004, 118:277-279.

5. Yang J, Weinberg RA: Epithelial-mesenchymal transition: at the crossroads of development and tumor metastasis. Dev Cell 2008, 14:818-829.

6. Yook JI, Li XY, Ota I, Hu C, Kim HS, Kim NH, Cha SY, Ryu JK, Choi YJ, Kim J, Fearon ER, Weiss SJ: A Wnt-Axin2-GSK3beta cascade regulates Snail1 activity in breast cancer cells. Nat Cell Biol 2006, 8:1398-1406.

7. Nieto MA: The snail superfamily of zinc-finger transcription factors. Nat Rev Mol Cell Biol 2002, 3:155-166.

8. Batlle E, Sancho E, Francí C, Domínguez D, Monfar M, Baulida J, García De Herreros A: The transcription factor snail is a repressor of E-cadherin gene expression in epithelial tumour cells. Nat Cell Biol 2000, 2:84-89.

9. Cano A, Pérez-Moreno MA, Rodrigo I, Locascio A, Blanco MJ, del Barrio MG, Portillo F, Nieto MA: The transcription factor snail controls epithelialmesenchymal transitions by repressing E-cadherin expression. Nat Cell Biol 2000, 2:76-83

10. Jin H, Yu Y, Zhang T, Zhou X, Zhou J, Jia L, Wu Y, Zhou BP, Feng Y: Snail is critical for tumor growth and metastasis of ovarian carcinoma. Int $J$ Cancer 2010, 126:2102-2111.

11. Yanagawa J, Walser TC, Zhu LX, Hong L, Fishbein MC, Mah V, Chia D, Goodglick L, Elashoff DA, Luo J, Magyar CE, Dohadwala M, Lee JM, St John MA, Strieter RM, Sharma S, Dubinett SM: Snail promotes CXCR2 liganddependent tumor progression in non-small cell lung carcinoma. Clin Cancer Res 2009, 15:6820-6829.

12. Kosaka T, Kikuchi E, Mikami S, Miyajima A, Shirotake S, Ishida M, Okada Y, Oya M: Expression of snail in upper urinary tract urothelial carcinoma: prognostic significance and implications for tumor invasion. Clin Cancer Res 2010, 16:5814-5823.

13. Yang MH, Chen CL, Chau GY, Chiou SH, Su CW, Chou TY, Peng WL, Wu JC: Comprehensive analysis of the independent effect of twist and snail in 
promoting metastasis of hepatocellular carcinoma. Hepatology 2009, 50:1464-1474.

14. Kim MA, Lee HS, Lee HE, Kim JH, Yang HK, Kim WH: Prognostic importance of epithelial-mesenchymal transition-related protein expression in gastric carcinoma. Histopathology 2009, 54:442-451.

15. Ryu HS, do Park J, Kim HH, Kim WH, Lee HS: Combination of epithelialmesenchymal transition and cancer stem cell-like phenotypes has independent prognostic value in gastric cancer. Hum Pathol 2012, 43:520-528.

16. Livak KJ, Schmittgen TD: Analysis of relative gene expression data using real-time quantitative PCR and the 2(-Delta DeltaC(T)) Method. Methods 2001, 25:402-408.

17. Kim WH, Park CK, Kim YB, et al: A standardized pathology report for gastric cancer. Korean J Pathol 2005, 39:106-113.

18. Japanese Gastric Cancer Association: Japanese classification of gastric carcinoma: 3rd English edition. Gastric Cancer 2011, 14:101-112.

19. Edge SB, Byrd DR, Compton CC, Fritz AG, Greene FL, Trotti A (Eds): American Joint Committee on Cancer Staging Manual. 7th edition. New York: Springer; 2009 .

20. Eisen MB, Spellman PT, Brown PO, Botstein D: Cluster analysis and display of genome-wide expression patterns. Proc Natl Acad Sci USA 1998, 95:14863-14868.

21. Lehner F, Kulik U, Klempnauer J, Borlak J: The hepatocyte nuclear factor 6 (HNF6) and FOXA2 are key regulators in colorectal liver metastases. FASEB J 2007, 21:1445-1462.

22. Wagstaff $L$, Kelwick R, Decock J, Edwards DR: The roles of ADAMTS metalloproteinases in tumorigenesis and metastasis. Front Biosci 2011, 16:1861-1872.

23. Kamai T, Yanai Y, Arai K, Abe H, Yamanishi T, Kurimoto M, Yoshida K: Increased interferon alpha receptor 2 mRNA levels is associated with renal cell carcinoma metastasis. BMC Cancer 2007, 7:159.

24. Ohtaki Y, Ishii G, Nagai K, Ashimine S, Kuwata T, Hishida T, Nishimura M, Yoshida J, Takeyoshi I, Ochiai A: Stromal macrophage expressing CD204 is associated with tumor aggressiveness in lung adenocarcinoma. J Thorac Oncol 2010, 5:1507-1515

25. Zhu Y, Bujo H, Yamazaki H, Ohwaki K, Jiang M, Hirayama S, Kanaki T, Shibasaki M, Takahashi K, Schneider WJ, Saito Y: LR11, an LDL receptor gene family member, is a novel regulator of smooth muscle cell migration. Circ Res 2004, 94:752-758.

26. Guaita S, Puig I, Franci C, Garrido M, Dominguez D, Batlle E, Sancho E, Dedhar S, De Herreros AG, Baulida J: Snail induction of epithelial to mesenchymal transition in tumor cells is accompanied by MUC1 repression and ZEB1 expression. J Biol Chem 2002, 277:39209-39216.

27. Miyoshi A, Kitajima Y, Kido S, Shimonishi T, Matsuyama S, Kitahara K, Miyazaki K: Snail accelerates cancer invasion by upregulating MMP expression and is associated with poor prognosis of hepatocellular carcinoma. Br J Cancer 2005, 92:252-258.

28. Peinado H, Marin F, Cubillo E, Stark HJ, Fusenig N, Nieto MA, Cano A: Snail and $\mathrm{E} 47$ repressors of $\mathrm{E}$-cadherin induce distinct invasive and angiogenic properties in vivo. J Cell Sci 2004, 117:2827-2839.

29. Côme C, Magnino F, Bibeau F, De Santa Barbara P, Becker KF, Theillet C, Savagner P: Snail and slug play distinct roles during breast carcinoma progression. Clin Cancer Res 2006, 12:5395-5402.

30. Rosivatz E, Becker KF, Kremmer E, Schott C, Blechschmidt K, Höfler H, Sarbia M: Expression and nuclear localization of Snail, an E-cadherin repressor, in adenocarcinomas of the upper gastrointestinal tract. Virchows Arch 2006, 448:277-287.

31. He H, Chen W, Wang X, Wang C, Liu F, Shen Z, Xu J, Gu J, Sun Y: Snail is an independent prognostic predictor for progression and patient survival of gastric cancer. Cancer Sci 2012, 103:1296-1303.

doi:10.1186/1471-2407-12-521

Cite this article as: Shin et al:: Overexpression of Snail is associated with lymph node metastasis and poor prognosis in patients with gastric cancer. BMC Cancer 2012 12:521.

\section{Submit your next manuscript to BioMed Central and take full advantage of:}

- Convenient online submission

- Thorough peer review

- No space constraints or color figure charges

- Immediate publication on acceptance

- Inclusion in PubMed, CAS, Scopus and Google Scholar

- Research which is freely available for redistribution

Submit your manuscript at www.biomedcentral.com/submit
( BioMed Central 